\title{
Elaboração e validação de escala diagramática para avaliação da severidade da ferrugem branca do crisântemo
}

\author{
Maria Angélica G. Barbosa ${ }^{1}$, Sami J. Michereff², Gustavo Mora-Aguilera ${ }^{3}$
}

\begin{abstract}
${ }^{1}$ Colegiado de Ciências Agrárias, Campus de Altamira, Universidade Federal do Pará, <angelguim @ig.com.br>. Bolsista do CNPq; ${ }^{2}$ Departamento de Agronomia - Área de Fitossanidade, Universidade Federal Rural de Pernambuco. Bolsista de Produtividade em Pesquisa do CNPq; ${ }^{3}$ Instituto de Fitosanidad, Colegio de Postgraduados, Montecillo, México. <morag@colpos.mx>
\end{abstract}

Autor para correspondência: Sami J. Michereff.<sami@ufrpe.br>

Data de chegada: 26/09/04. Aceito para publicação em: 23/06/05.

\begin{abstract}
Barbosa, M.A.G.; Michereff, S.J.; Mora-Aguilera, G. Elaboration and validation of diagrammatic key to evaluate white rust severity in Chrysanthemum. Summa Phytopathologica, v.32, p. 57-62, 2006.

White rust, caused by Puccinia horiana, is considered the major disease of Chrysanthemum in Brazil, which results in severe losses for the growers. Despite of its economic importance, very few epidemiological studies have been carried out in Brazil. In order to conduct these studies it is necessary to develop standard methods to quantify disease severity under field conditions. Therefore, a diagrammatic key, including the levels $1,3,6,10,18$, and $30 \%$ of diseased leaf area, was elaborated. The severity of the white rust was estimates using the key or not in order to test its accuracy, precision and reproducibility. The diagrammatic key was validated by 10 raters using 50 leaves with different levels of severity, which were previously measured by the AutoCAD ${ }^{\circledast}$ software. Two evaluations were performed

with the key at 7-day intervals when different sequences of the same leaves were visually estimated by the same raters. The accuracy and precision of each rater were determined by using simple linear regression between actual and estimated severity. When the key was not used, all raters overestimated disease severity indicating the presence of constant positive errors for all levels of disease severity. The evaluations using the diagrammatic key were more accurate for the great majority of the raters and more precise for all the raters. In addition, the key also showed good repeatability and high level of reproducibility among the evaluations from the different raters. The diagrammatic key is suitable for the evaluation of severity of white rust of Chrysanthemum.
\end{abstract}

Additional keywords: Dendranthema grandiflora, Puccinia horiana, pathometry.

\section{RESUMO}

Barbosa, M.A.G.; Michereff, S.J.; Mora-Aguilera, G. Elaboração e validação de escala diagramática para avaliação da severidade da ferrugem branca do crisântemo. Summa Phytopathologica, v.32, p. 57-62, 2006.

A ferrugem branca, causada pelo fungo Puccinia horiana, é considerada a principal doença do crisântemo no Brasil, induzindo severas perdas aos produtores. Apesar da importância, inexistem estudos epidemiológicos no país, e para que estes estudos sejam realizados, é necessário o desenvolvimento de métodos padronizados de quantificação da severidade da doença no campo. Visando atender a essa demanda, foi elaborada uma escala diagramática com os níveis de 1 , $3,6,10,18$ e $30 \%$ de área foliar lesionada, testando-se a acurácia, a precisão e a reprodutibilidade das estimativas de severidade da ferrugem branca com e sem a sua utilização. Na validação da escala diagramática, 50 folhas com diferentes níveis de severidade da doença, mensurados previamente com o programa AutoCADâ, foram avaliadas por 10 pessoas sem e com a utilização da escala diagramática. Foram realizadas duas avaliações com a utilização da escala, com intervalo de sete dias, onde seqüências diferentes das mesmas folhas foram estimadas visualmente pelos mesmos avaliadores. A acurácia e a precisão de cada avaliador foi determinada por regressão linear simples, entre a severidade real e a estimada. Sem o auxílio da escala, todos os avaliadores superestimaram consistentemente a severidade, indicando a presença de desvios positivos constantes para todos os níveis de severidade da doença. As avaliações realizadas com a escala diagramática foram mais acuradas nas estimativas da maioria dos avaliadores e mais precisas para todos os avaliadores, além de proporcionar boa repetibilidade e elevada reprodutibilidade entre avaliações de diferentes avaliadores. A escala diagramática mostrou-se adequada para avaliação da severidade da ferrugem branca do crisântemo.

Palavras-chave adicionais: Dendranthema grandiflora, Puccinia horiana, patometria. 
A ferrugem branca, causada pelo fungo Puccinia horiana Henn., é considerada a principal doença do crisântemo (Dendranthema grandiflora Tzvelev) no país $(4,6,7)$. Os sintomas da ferrugem branca apresentam-se, inicialmente, na forma de pequenas manchas branco-amareladas na face superior das folhas, assumindo posteriormente uma coloração marrom-escura na parte central das lesões. À medida que a infecção progride, surgem na face inferior das folhas, nas áreas correspondentes às manchas amareladas, pústulas salientes, esbranquiçadas a rosadas, cerosas, circulares e proeminentes, que consistem as estruturas reprodutivas do patógeno, principalmente os teliosporos e basidiosporos. Quando ocorre a coalescência das pústulas, as folhas podem secar e cair, reduzindo drasticamente a área fotossintetizante da planta $(4,6)$. A doença diminui sensivelmente a quantidade e a qualidade das flores produzidas, sendo que as perdas variam com a suscetibilidade das variedades e o estádio de desenvolvimento das plantas $(17,21)$.

Embora a ferrugem branca do crisântemo possa induzir perdas severas aos produtores (6), inexistem estudos epidemiológicos dessa doença no Brasil. Esses estudos requerem métodos padronizados para quantificação da severidade (porcentagem ou proporção de área foliar lesionada) da doença no campo, motivo pelo qual o desenvolvimento de métodos de quantificação pode prevenir sérios erros na aquisição de dados (11).

Considerando que a ferrugem branca do crisântemo é uma doença foliar, sua intensidade é melhor expressa pela determinação da severidade. Como a severidade das doenças é estimada visualmente, está sujeita a grande subjetividade e pode induzir a graves erros de acurácia, precisão e reprodutibilidade (24). A acurácia refere-se à proximidade de uma estimativa a um valor real de quantidade de doença avaliada, a precisão refere-se à confiabilidade e/ou repetibilidade associadas com uma estimativa e a reprodutibilidade refere-se à ausência de variação estatística significativa nas estimativas quando a mesma amostra de doença é avaliada por outro avaliador (3).

A avaliação da severidade da ferrugem branca do crisântemo tem sido realizada com o auxílio de escalas descritivas de notas $(7,17,21)$, estimativas diretas da área foliar coberta por pústulas (20) e contagem do número de pústulas por folha (12). Considerando que os procedimentos de quantificação de doenças necessitam ser de fácil e rápida utilização para uma ampla gama de condições, mas também propiciar resultados acurados, precisos e reprodutíveis (3), os métodos utilizados na quantificação da severidade da ferrugem branca do crisântemo apresentam várias limitações, motivo pelo qual o uso de uma escala diagramática pode constituir excelente opção.

Escalas diagramáticas têm sido ferramentas muito úteis para minimizar a subjetividade das estimativas de severidade e também auxiliar o avaliador. Entretanto, devem ser de fácil uso, aplicáveis em diferentes condições e produzir resultados reprodutíveis, além de apresentarem níveis suficientes que representem todos os estádios de desenvolvimento da doença (2).

Considerando-se a inexistência de métodos padronizados para quantificação da ferrugem branca do crisântemo, este trabalho teve como objetivos desenvolver uma escala diagramática para avaliação da severidade da doença e analisar os níveis de acurácia, precisão e reprodutibilidade das estimativas geradas com sua utilização.

\section{MATERIALEMÉTODOS}

\section{Elaboração da escala diagramática}

Para elaboração da escala diagramática foram coletadas 200 folhas de crisântemo (var. Calábria) em plantios comerciais no município de Gravatá - PE, com diferentes níveis de severidade da ferrugem branca. As folhas foram reproduzidas por fotocópias coloridas e digitalizadas, e com auxílio do programa AutoCAD ${ }^{\circledR}$ (Autodesk, 1998) foram determinadas a área foliar total e a área lesionada de cada folha, obtendo-se a severidade da doença. Utilizando o valor máximo de severidade da doença constatada nas folhas coletadas, os intervalos da escala foram calculados com o auxílio do programa 2LOG (24) e os valores obtidos arredondados para números inteiros. Baseando-se na lei de Weber-Fechner de acuidade visual (10), bem como na forma, distribuição e freqüência das lesões, foi confeccionada uma escala diagramática logarítmica com seis níveis de severidade.

\section{Validação da escala diagramática}

No teste de validação da escala diagramática foram utilizadas fotocópias coloridas de 50 folhas de crisântemo com sintomas de ferrugem branca apresentando diferentes níveis de severidade. A severidade da doença foi avaliada por 10 pessoas, a maioria sem experiência na quantificação de doenças. Inicialmente, a severidade foi estimada sem o auxílio da escala diagramática e, após sete dias, com o auxílio dessa. Visando avaliar a repetibilidade das estimativas com a escala diagramática, 14 dias após a primeira avaliação, nova sequiência das mesmas folhas foi organizada e uma segunda estimativa visual com auxílio da escala foi efetuada pelos mesmos avaliadores.

A acurácia e a precisão de cada avaliador foi determinada por meio de regressão linear simples, com a severidade real obtida eletronicamente como variável independente e a severidade estimada pelo avaliador como variável dependente. A acurácia das estimativas de cada avaliador e do conjunto de avaliadores foi determinada pelo teste $t$ aplicado ao intercepto da regressão line$\operatorname{ar}(a)$, para verificar a hipótese Ho: $a=0$, e ao coeficiente angular da reta $(b)$, para testar a hipótese Ho: $b=1$, ao nível $5 \%$ de probabilidade. Valores de intercepto significativamente diferentes de 0 indicam a presença de desvios constantes, enquanto valores de coeficiente angular da reta que diferem significativamente de 1 indicam a presença de desvios sistemáticos. A precisão das estimativas foi obtida pelo coeficiente de determinação da regressão $\left(R^{2}\right)$, pelos erros absolutos (severidade estimada menos real) e pela repetibilidade das estimativas, determinada pela regressão da segunda avaliação em relação à primeira de uma mesma unidade amostral. A reprodutibilidade das estimativas foi determinada pelos valores de $R^{2}$ obtidos de regressões lineares entre as severidades estimadas por diferentes avaliadores combinados em pares $(3,11,18)$. As análises de regressão foram efetuadas com o auxílio do programa Microsoft Excel $2000^{\circledR}$ (Microsoft Corporation, 2000).

\section{RESULTADOS E DISCUSSÃO}

O valor máximo de severidade da ferrugem branca nas folhas de crisântemo coletadas nos plantios comerciais foi de $29,95 \%$. Valores acima de $30 \%$ são raramente encontrados no campo, pois causam queda prematura da folha. A escala diagramática para quantificação da ferrugem branca do crisântemo foi desenhada 
com seis níveis de severidade, representados pelos valores de 1, 3, 6, 10, 18 e 30\% de área foliar lesionada (Figura 1).

Sem a utilização da escala diagramática, os avaliadores foram pouco acurados, pois $90 \%$ apresentaram valores do intercepto significativamente diferentes de zero $(\mathrm{P} \leq 0,05)$ para as retas de regressão entre severidade real e estimada, com valor médio de 4,07 (Tabela 1). Todos esses avaliadores superestimaram consistentemente a severidade, indicando a presença de desvios positivos constantes para todos os níveis de severidade da doença. Com a utilização da escala diagramática, a maioria dos avaliadores melhorou os níveis de acurácia das estimativas de severidade (Tabela 1). Nas duas avaliações com o auxílio da escala, somente $30 \%$ dos avaliadores (B, E e I) apresentaram valores do intercepto significativamente diferentes de zero $(\mathrm{P} \leq 0,05)$, todos com desvios positivos constantes. Os valores médios do intercepto na primeira $(1,01)$ e na segunda $(1,15)$ avaliação não diferiram significativamente de zero $(\mathrm{P} \leq 0,05)$ (Tabela 1$)$, indicando a redução dos erros verificados sem a utilização da escala. Em relação aos valores do coeficiente angular da reta, $60 \%$ dos avaliadores (B, $\mathrm{D}, \mathrm{F}, \mathrm{H}, \mathrm{I}$ e J) apresentaram valores significativamente diferentes de $1(\mathrm{P} \leq 0,05)$ sem a utilização da escala diagramática, indicando a presença de desvios sistemáticos (Tabela 1). Com a utilização da escala, $90 \%$ dos avaliadores na primeira e $100 \%$ na segunda avaliação apresentaram coeficientes angulares significativamente similares de $1(\mathrm{P} \leq 0,05)$ (Tabela 1$)$, indicando a redução significativa dos erros sistemáticos das estimativas.

A precisão das estimativas sem o auxílio da escala também foi baixa, pois os coeficientes de determinação variaram entre 0,59 e 0,77 , com média de 0,68 . Os erros na quantificação da severidade, representados pelas diferenças entre os valores estimados visualmente e os reais obtidos eletronicamente, reduzi-
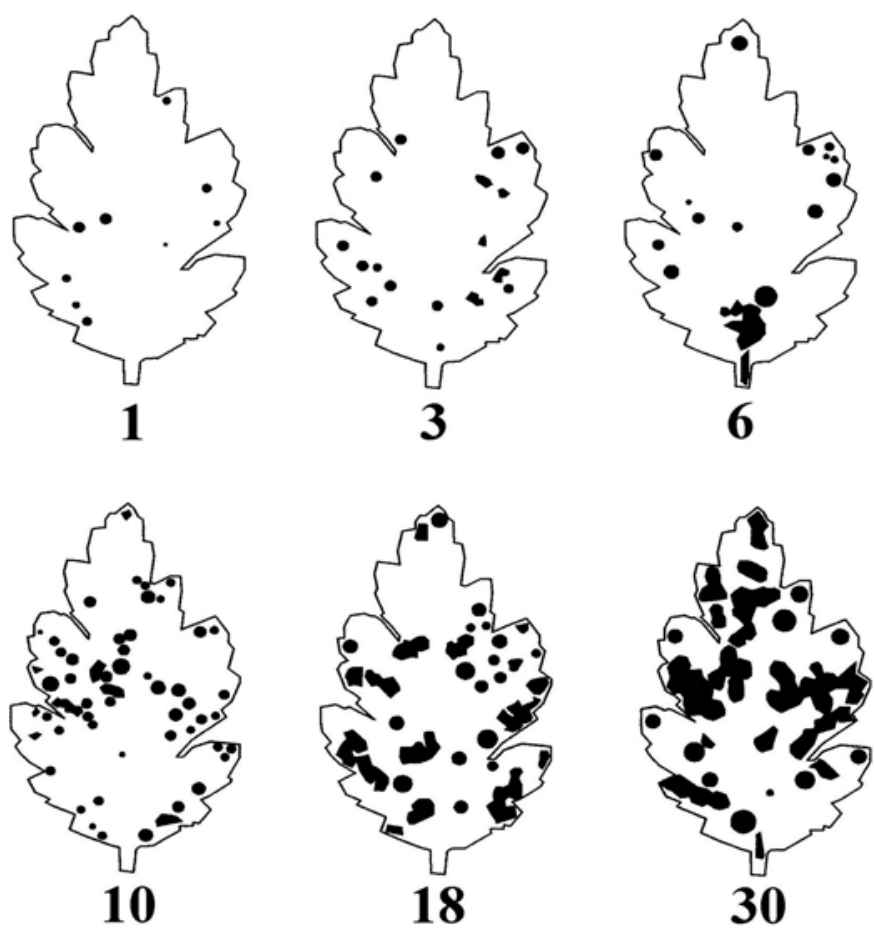

Figura 1. Escala diagramática para avaliação da ferrugem branca do crisântemo indicando os níveis de 1, 3, 6, 10, 18 e 30\% de severidade.

Tabela 1. Intercepto $(a)$, coeficiente angular da reta $(b)$ e coeficiente de determinação $\left(R^{2}\right)$ de equações de regressão linear simples relacionando estimativas visuais da severidade da ferrugem branca do crisântemo efetuadas por avaliadores, sem e com o auxílio da escala diagramática, à severidade real determinada eletronicamente.

\begin{tabular}{|c|c|c|c|c|c|c|c|c|c|}
\hline \multirow{3}{*}{ Avaliador } & \multicolumn{3}{|c|}{ Sem Escala } & \multicolumn{6}{|c|}{ Com Escala } \\
\hline & \multirow[b]{2}{*}{$\mathrm{a}$} & \multirow[b]{2}{*}{ b } & \multirow[b]{2}{*}{$\mathrm{R}^{2}$} & \multicolumn{3}{|c|}{$1^{\mathrm{a}}$ avaliação } & \multicolumn{3}{|c|}{$2^{\mathrm{a}}$ avaliação } \\
\hline & & & & $\mathrm{a}$ & $\mathrm{b}$ & $\mathrm{R}^{2}$ & $\mathrm{a}$ & $\mathrm{b}$ & $\mathrm{R}^{2}$ \\
\hline A & 0,94 & 1,04 & 0,77 & $-0,07$ & $0,79 *$ & 0,87 & $-0,98$ & 1,05 & 0,90 \\
\hline B & $4,47^{*}$ & $1,21^{*}$ & 0,76 & $2,74 *$ & 1,00 & 0,85 & $3,75^{*}$ & 0,99 & 0,90 \\
\hline $\mathrm{C}$ & $5,83^{*}$ & 0,82 & 0,65 & 1,49 & 0,90 & 0,84 & 1,38 & 0,89 & 0,87 \\
\hline $\mathrm{D}$ & $4,30^{*}$ & $1,56^{*}$ & 0,71 & $-0,02$ & 0,92 & 0,84 & $-0,10$ & 1,01 & 0,88 \\
\hline $\mathrm{E}$ & $4,75^{*}$ & 1,13 & 0,70 & $1,97^{*}$ & 0,97 & 0,82 & $2,55^{*}$ & 0,91 & 0,86 \\
\hline $\mathrm{F}$ & $2,47^{*}$ & $1,50^{*}$ & 0,66 & 0,31 & 1,02 & 0,91 & 1,16 & 1,02 & 0,91 \\
\hline G & $6,40^{*}$ & 1,10 & 0,70 & 1,38 & 0,95 & 0,87 & 0,87 & 0,96 & 0,88 \\
\hline $\mathrm{H}$ & $2,41^{*}$ & $1,21^{*}$ & 0,61 & 0,50 & 1,00 & 0,86 & 0,24 & 0,98 & 0,88 \\
\hline I & $6,91 *$ & $2,03 *$ & 0,64 & $2,19 *$ & 0,86 & 0,76 & $2,61 *$ & 0,90 & 0,85 \\
\hline $\mathrm{J}$ & $2,26^{*}$ & $1,53^{*}$ & 0,59 & $-0,44$ & 0,92 & 0,80 & 0,03 & 0,90 & 0,84 \\
\hline Média & $4,07 *$ & $1,31^{*}$ & 0,68 & 1,01 & 0,93 & 0,84 & 1,15 & 0,96 & 0,88 \\
\hline
\end{tabular}

*Asterisco indica que a hipótese de nulidade $(a=0$ ou $b=1)$ foi rejeitada pelo teste $t(\mathrm{P}=0,05)$. 
ram drasticamente com a utilização da escala diagramática. A distribuição dos resíduos das avaliações realizadas sem o auxílio da escala diagramática apresentou estimativas com erros variando entre -9,93 e 59,81 (Figura 2A). Por outro lado, com a utilização da escala os erros não apresentaram um padrão indesejável definido e variaram na primeira avaliação entre -12,18 e 11,87 (Figura 2B), enquanto na segunda avaliação entre -8,92 e 12,54 (Figura 2C). A maioria dos erros absolutos dos avaliadores que utilizaram a escala foi inferior a $10 \%$, considerado bom segundo os critérios adotados em vários estudos de avaliação de escalas diagramáticas $(1,5,8,9,13,14,15,16,23)$. A presença de algum nível de erro absoluto nas mensurações pode ser compensada pela rapidez e padronização que resultam do uso de escalas diagramáticas. Além disso, como na maioria dos métodos de quantificação de severidade de doenças, o uso de escalas diagramáticas está sujeito a um certo grau de subjetividade, o que pode ser minimizado com o treinamento dos avaliadores $(8,15,19)$.

Todos os avaliadores melhoraram a precisão das estimativas com a utilização da escala, com coeficientes de determinação variando entre 0,76 e 0,91 na primeira avaliação, e entre 0,84 e 0,91 na segunda, com médias respectivas de 0,84 e 0,88 (Tabela 1), assemelhando-se ao verificados em outros estudos de validação de escalas $(5,9,14,16,24)$. A maioria dos avaliadores melhorou a precisão das estimativas na segunda avaliação com a escala em relação à primeira.

A diferença entre os avaliadores na mensuração da ferrugem branca do crisântemo era esperada, pois a qualidade da estimativa da doença, além de ser influenciada por estímulos e respostas psicológicas, pode ser afetada por fatores como complexidade da unidade amostral, tamanho e forma das lesões, cor e número de lesões na unidade amostral (11), fadiga, dificuldade de concentração na tarefa (22) e experiência de cada indivíduo na avaliação da doença $(15,23)$.

Em geral, os avaliadores apresentaram boa repetibilidade nas estimativas com o uso da escala diagramática, pois a quantidade média de variação na primeira avaliação explicada pela segunda avaliação foi de $82 \%$ (Tabela 2), assemelhando-se ao constatado por Nutter et al. (18) na mensuração da mancha foliar de Agrostis palustris Huds., causada por Sclerotinia homoeocarpa Bennett. Para 50\% dos avaliadores os valores do intercepto foram significativamente diferentes de zero $(\mathrm{P} \leq 0,05)$ entre as duas avaliações, enquanto para $70 \%$ os valores do coeficiente angular da reta não foram significativamente diferentes de $1(\mathrm{P} \leq 0,05)$ (Tabela 2), indicando coincidência em precisão. Além disso, os valores médios do intercepto e do coeficiente angular da reta não diferiram significativamente de zero e $1(\mathrm{P} \leq 0,05)$, respectivamente.

A reprodutibilidade das avaliações da severidade sem a utilização da escala diagramática foi baixa, pois as regressões lineares entre as severidades estimadas pelos 10 avaliadores produziram coeficientes de determinação variando de 0,32 a 0,84 , sendo que em somente $2,2 \%$ dos casos foi $\geq 0,75 \%$ (Tabela 3 ). Por outro lado, com a utilização da escala diagramática as avaliações apresentaram elevada reprodutibilidade, pois nas duas avaliações os valores do coeficiente de determinação foram elevados,

Tabela 2. Intercepto (a), coeficiente angular da reta $(b)$ e coeficiente de determinação $\left(R^{2}\right)$ de equações de regressão linear simples relacionando a segunda à primeira estimativa da ferrugem branca do crisântemo por um mesmo avaliador, com o auxílio da escala diagramática.

\begin{tabular}{cccc}
\hline Avaliador & $a$ & $b$ & $R^{2}$ \\
\hline A & 0,17 & $1,20^{*}$ & 0,84 \\
B & $2,27^{*}$ & 0,90 & 0,86 \\
C & 0,93 & 0,90 & 0,84 \\
D & 0,87 & 0,99 & 0,86 \\
E & $2,31^{*}$ & $0,80^{*}$ & 0,76 \\
F & $1,79^{*}$ & 0,91 & 0,84 \\
G & 0,22 & 0,95 & 0,88 \\
H & 0,67 & 0,89 & 0,85 \\
I & $2,42^{*}$ & 0,86 & 0,74 \\
J & $2,00^{*}$ & $0,81 *$ & 0,71 \\
\hline Média & 1,36 & 0,92 & 0,82 \\
\hline
\end{tabular}

*Asterisco indica que a hipótese de nulidade $(a=0$ ou $b=1)$ foi rejeitada pelo teste $t(\mathrm{P}=0,05)$.
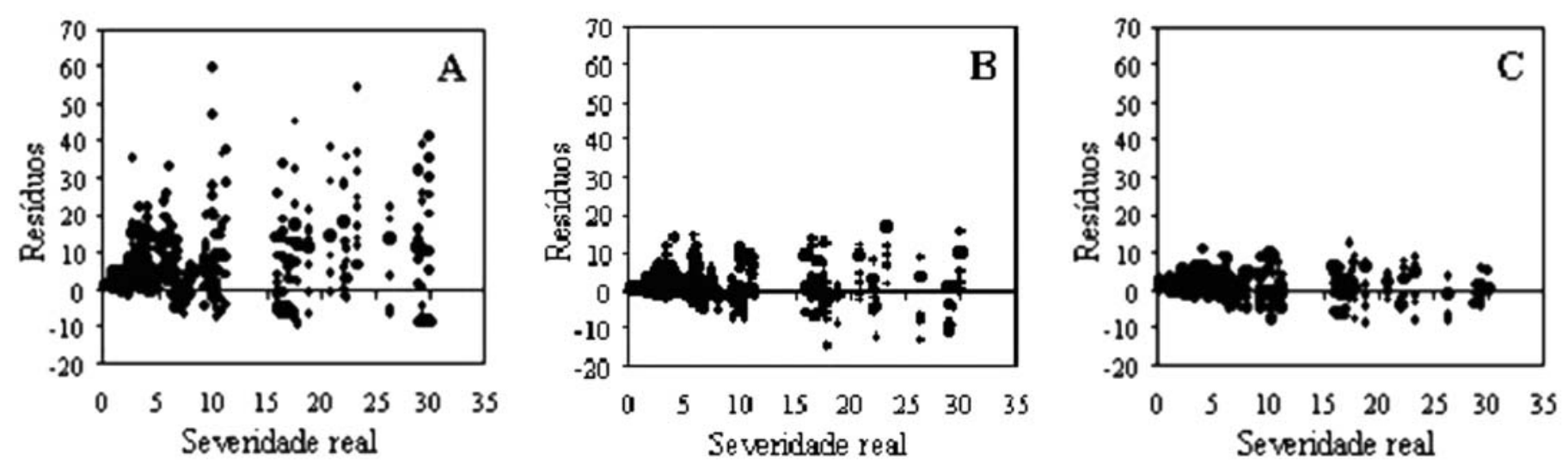

Figura 2. Resíduos (severidade estimada - severidade real) das estimativas da ferrugem branca do crisântemo realizadas sem o auxílio da escala diagramática (A) e com a escala diagramática na primeira (B) e segunda (C) avaliação. 
variando entre 0,67 a 0,92 , sendo $\geq 0,75$ em $80 \%$ dos casos na primeira avaliação e em $91,1 \%$ dos casos na segunda (Tabela 3 ), assemelhado-se ao constado na validação de escalas diagramáticas para outros patossistemas $(5,8,13,14,20)$. O uso da escala diagramática aumentou a reprodutibilidade das estimativas obtidas por diferentes avaliadores, e do ponto de vista prático, diferentes experimentos, conduzidos por avaliadores distintos, serão comparáveis desde que utilizem a escala diagramática.
A escala diagramática proposta para avaliação da severidade da ferrugem branca do crisântemo mostrou ser de fácil utilização, capaz de fornecer uma rápida estimativa da doença e proporcionar boa acurácia, precisão e reprodutibilidade das estimativas. Portanto, pode ser utilizada em levantamentos de campo, estudos de progresso e disseminação da doença, bem como na avaliação da resistência quantitativa de germoplasmas de crisântemo e de outras medidas de controle da doença.

Tabela 3. Coeficiente de determinação $\left(R^{2}\right)$ de equações de regressão linear simples relacionando as estimativas da ferrugem branca do crisântemo entre avaliadores sem o auxílio da escala diagramática e com a escala diagramática na primeira e segunda avaliação.

\begin{tabular}{|c|c|c|c|c|c|c|c|c|c|}
\hline \multicolumn{10}{|c|}{ Sem Escala } \\
\hline Avaliador & $\mathrm{B}$ & $\mathrm{C}$ & $\mathrm{D}$ & $\mathrm{E}$ & $\mathrm{F}$ & G & $\mathrm{H}$ & I & $\mathrm{J}$ \\
\hline A & 0,73 & 0,62 & 0,69 & 0,61 & 0,57 & 0,61 & 0,43 & 0,52 & 0,54 \\
\hline B & & 0,73 & 0,63 & 0,53 & 0,56 & 0,54 & 0,59 & 0,56 & 0,63 \\
\hline $\mathrm{C}$ & & & 0,63 & 0,50 & 0,53 & 0,56 & 0,53 & 0,60 & 0,52 \\
\hline D & & & & 0,65 & 0,60 & 0,60 & 0,60 & 0,73 & 0,58 \\
\hline $\mathrm{E}$ & & & & & 0,50 & 0,73 & 0,32 & 0,51 & 0,36 \\
\hline F & & & & & & 0,58 & 0,69 & 0,65 & 0,51 \\
\hline G & & & & & & & 0,38 & 0,52 & 0,48 \\
\hline $\mathrm{H}$ & & & & & & & & 0,84 & 0,65 \\
\hline I & & & & & & & & & 0,66 \\
\hline
\end{tabular}

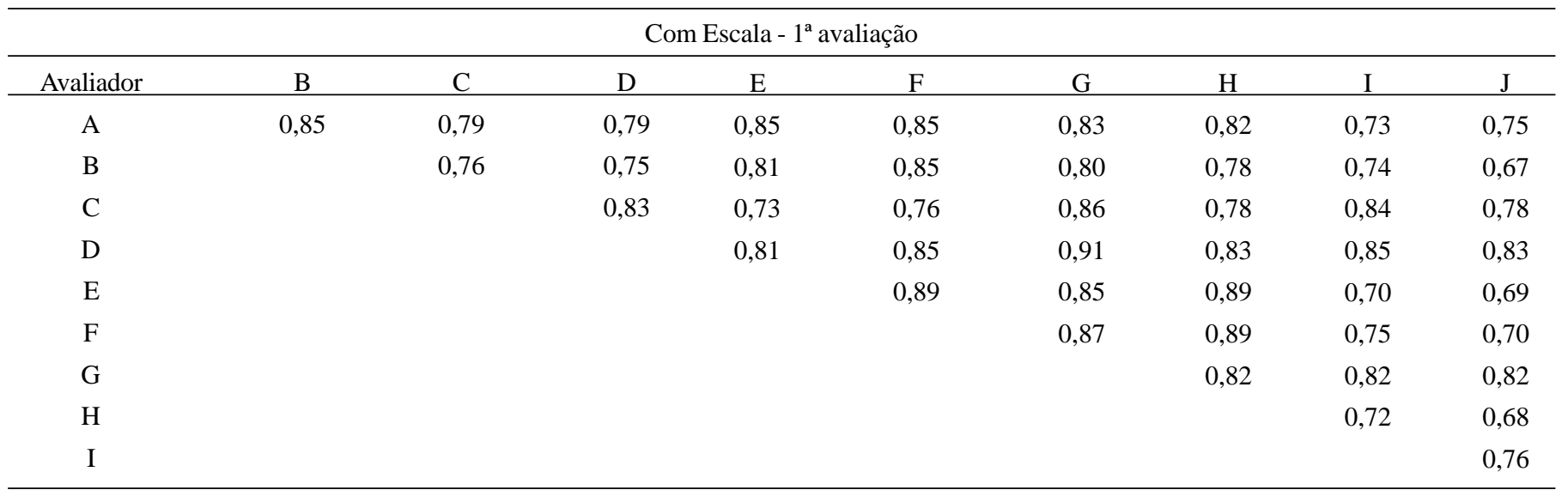

\begin{tabular}{|c|c|c|c|c|c|c|c|c|c|}
\hline \multicolumn{10}{|c|}{ Com Escala - $2^{\mathrm{a}}$ avaliação } \\
\hline A & 0,82 & 0,83 & 0,76 & 0,77 & 0,80 & 0,79 & 0,76 & 0,77 & 0,74 \\
\hline $\mathrm{C}$ & & & 0,75 & 0,77 & 0,83 & 0,85 & 0,85 & 0,78 & 0,73 \\
\hline $\mathrm{D}$ & & & & 0,82 & 0,85 & 0,84 & 0,84 & 0,81 & 0,76 \\
\hline $\mathrm{F}$ & & & & & & 0,81 & 0,92 & 0,82 & 0,84 \\
\hline G & & & & & & & 0,84 & 0,82 & 0,73 \\
\hline $\mathrm{H}$ & & & & & & & & 0,82 & 0,82 \\
\hline I & & & & & & & & & 0,75 \\
\hline
\end{tabular}




\section{AGRADECIMENTOS}

Os autores expressam seus agradecimentos a todos os avaliadores que participaram das etapas de validação da escala diagramática.

\section{REFERÊNCIAS BIBLIOGRÁFICAS}

1. Amorim, L.; Bergamin Filho, A.; Palazzo, D.A.; Bassanezi, R.B.; Godoy, C.V.; Torres, G.M. Clorose variegada dos citros: uma escala diagramática para avaliação da severidade da doença. Fitopatologia Brasileira, Brasília, v.18, n.2, p.174-180, 1993.

2. Bergamin Filho, A.; Amorim, L. Doenças de plantas tropicais: epidemiologia e controle econômico. São Paulo: Agronômica Ceres, 1996. 299p.

3. Campbell, C.L.; Madden, L.V. Introduction to plant disease epidemiology. New York: John Wiley, 1990. 532p.

4. Coutinho, L.N.; Russomano, O.M.R.; Toföli, J.G.; Domingues, R.J.; Oliveira, S.H.F.; Figueiredo, M.B. Doenças fúngicas. In: Imenes, S.D.L.; Alexandre, M.A.V. (Ed.) Aspectos fitossanitários do crisântemo. São Paulo: Instituto Biológico, 1996. p.23-34. (Boletim Técnico, 5).

5. Díaz, C.G.; Bassanezi, R.B.; Bergamin Filho, A. Desenvolvimento e validação de uma escala diagramática para Xanthomonas axonopodis pv. phaseoli em feijoeiro. Summa Phytopathologica, Jaboticabal, v.27, n.1, p.35-39, 2001.

6. Freire, F.C.O.; Viana, F.M.P.; Lima, R.C.A. Ocorrência da ferrugem-branca-do-crisântemo no estado do Ceará. Fortaleza: Embrapa Agroindústria Tropical, 2002. 2p. (Comunicado Técnico, 75).

7. Freitas, J.C.; Agostini, L.A.; Matsuoka, S.; Caldari Jr., P.; Garcia, A.A.F.; Oliveira, S.H.F. Controle da ferrugem branca do crisântemo (Puccinia horiana) em condições de casas de vegetação com diferentes mantas de cobertura. Summa Phytopathologica, Jaboticabal, v.23, n.3/4, p.268-271, 1997.

8. Godoy, C.V.; Carneiro, S.M.T.P.G.; Iamuti, M.T.; Dalla Pria, M.; Amorim, L.; Berger, R.D.; Bergamin Filho, A. Diagramatic scale for bean: development and validation. Zeitschrift für Pflanzenkrankheiten und Pflanzenschutz, Stuttgart, v.104, n.4, p.336-345, 1997.

9. Gomes, A.M.A.; Michereff, S.J.; Mariano, R.L.R. Elaboração e validação de escala diagramática para cercosporiose da alface. Summa Phytopathologica, Botucatu, v.30, n.1, p.38-42, 2004.

10. Horsfall, J.G.; Cowling, E.B. Pathometry: the measurement of plant disease. In: Horsfall, J.G.; Cowling, E.B. (Ed.) Plant disease: an advanced treatise - how disease develops in populations. New York: Academic Press, 1978. v.2, p.119-136.

11. Kranz, J. Measuring plant disease. In: Kranz, J.; Rotem, J. (Ed.) Experimental techniques in plant disease epidemiology. Heidelberg: Springer-Verlag, 1988. p.35-50.

12. Lam, C.H.; Lim, T.K. Efficacy of hexaconazole for the control of white rust on chrysanthemum and powdery mildew on roses. In- ternational Journal of Pest Management, London, v.39, n.2, p.156-160, 1993.

13. Leite, R.M.V.B.C.; Amorim, L. Elaboração e validação de escala diagramática para mancha de Alternaria em girassol. Summa Phytopathologica, Botucatu, v. 28, n.1, p.14-19, 2002.

14. Martins, M.C.; Guerzoni, R.A.; Câmara, G.M.S.; Mattiazzi, P.; Lourenço, S.A.; Amorim, L. Escala diagramática para a quantificação do complexo de doenças foliares de final de ciclo em soja. Fitopatologia Brasileira, Fortaleza, v.29, n.2, p.179-184, 2004.

15. Michereff, S.J.; Maffia, L.A.; Noronha, M.A. Escala diagramática para avaliação da severidade da queima das folhas do inhame. Fitopatologia Brasileira, Brasília, v.25, n.4, p.612-619, 2000.

16. Michereff, S.J.; Pedrosa, R.A.; Noronha, M.A.; Martins, R.B.; Silva, F.V. Escala diagramática e tamanho de amostras para avaliação da severidade da mancha parda da mandioca (Cercosporidium henningsii). Agrotrópica, Itabuna, v.10, n.3, p.143-148, 1998.

17. Norman, M.T.; García, F.A.; Sandoval, R.F.R.; Vázquez, G.L.M.; Aquino, M.J.; Corona, R.M.C.; Pedral, M.E. Nivel de daño causado por Puccinia horiana P. Henn. en crisantemo (Dendrathema grandiflora Tzvelev). Fitopatología, Lima, v.31, n.2, p.133-139, 1996.

18. Nutter Jr., F.W.; Gleason, M.L.; Jenco, J.H.; Christians, N.C. Assessing the accuracy, intra-rater repeatability, and inter-rater reliability of disease assessment systems. Phytopathology, St. Paul, v.83, n.8, p.806-812, 1993.

19. Nutter Jr., F.W.; Schultz, P.M. Improving the accuracy and precision of disease assessments: selection of methods and use of computer-aided training programs. Canadian Journal of Plant Pathology, Ottawa, v.17, n.1, p.174-184, 1995.

20. Rodríguez-Navarro, J.A.; Zavaleta-Mejia, E.; Alatorre-Rosas, R. Epidemiología y manejo de la roya blanca (Puccinia horiana $\mathrm{P}$. Henn.) del crisantemo (Dendrathema grandiflora Tzvelev). Fitopatología, Lima, v.31, n.2, p.122-132, 1996.

21. Sandoval, R.F.R.; Norman, M.T.; Corona, R.M.C.; Aquino, M.J.; Vázquez, G.L.M.; García, F.A. Determinación del nivel de daño causado por Puccinia horiana Henn. en 15 variedades de crisantemo (Dendranthema grandiflora Tzvelev.) cultivadas en México. Fitopatología, Lima, v.32, n.3, p.160-165, 1997.

22. Shokes, F.M.; Berger, R.D.; Smith, D.H.; Rasp, J.M. Reliability of disease assessment procedures: a case study with late leafspot of peanut. Oléagineux, Paris, v.42, n.4, p.245-251, 1987.

23. Sposito, M.B.; Amorim, L.; Belasque Junior, J.; Bassanezi, R.B.; Aquino, R. Elaboração e validação de escala diagramática para avaliação da severidade da mancha preta em frutos cítricos. Fitopatologia Brasileira, Fortaleza, v.29, n.1, p.81-85, 2004.

24. Tovar-Soto, A.; Hernandez-Martínez, M.; Cristóbal-Alejo, J.; Romero-Hijo, R.; Mora-Aguilera, G. Escala logarítimica diagramática de severidad de la mancha negra (Colletotrichum gloeosporioides Penz.) en chirimoyo (Annona cherimola Mill). Revista Mexicana de Fitopatología, Sonora, v.20, n.1, p.103-109, 2002. 